\title{
Endocarditis Due to Listeria monocytogenes in an Academic Teaching Hospital: Case Report
}

\author{
Claudia Summa and Sandra A N Walker
}

\section{INTRODUCTION}

$\mathrm{E}$ ndocarditis is a rare but serious infection observed in about - $8 \%$ of patients infected with Listeria monocytogenes. ${ }^{1}$ According to Antolin and others, ${ }^{2}$ there had been 68 case reports up to 2008 . The reported mortality rate for this infection, which has ranged from $37 \%$ to $50 \%$, has decreased appreciably in recent decades. ${ }^{1}$

Listeria monocytogenes is an aerobic, gram-positive coccobacillus. The organism may be isolated from soil, dust, animal feed, water, sewage, and the tissues or fluids of almost any type of animal. It is frequently found in raw and unprocessed food products such as meats, vegetables, and dairy and delicatessen products that are intended for consumption without further heating. ${ }^{.}$Reported outbreaks of this organism have been associated with contaminated rice salad, coleslaw, soft cheese, hot dogs, shrimp, chocolate milk, and corn salad. ${ }^{1}$ In the summer of 2008, an outbreak of listeriosis associated with one company's meat products occurred across 7 provinces in Canada; a total of 56 people were affected, and at least 21 people died. ${ }^{3}$

Although $L$ monocytogenes is a transient colonizer in the human gastrointestinal tract, infection does not occur unless host factors promoting invasive disease are present or the amount of bacteria delivered to the intestinal tract is great enough to overwhelm local gastrointestinal barriers. ${ }^{4}$ This organism can produce severe sepsis, meningoencephalitis, focal infections in infants and adults, and death, as illustrated by the Canadian outbreak.

Listeria monocytogenes penetrates into a wide range of host cells (phagocytes, epithelial cells, and parenchymal cells) through phagocytosis or induced phagocytosis. ${ }^{5}$ A cluster of virulence genes enables this pathogenic bacterium to evade the phagocytic vacuoles, and it resides and multiplies within the cytoplasm. ${ }^{5}$ Once the organisms reach the cytoplasm, the bacteria wrap themselves in a sheet of actin filaments derived from the host cell, forming a comet-like structure. ${ }^{5}$ In essence, the bacteria become hidden within the host's cells, protected from the host's humoral defence mechanisms and from antibiotics in the extracellular fluid. ${ }^{5}$
In this article, we describe a case of endocarditis caused by $L$ monocytogenes and discuss the features of this disease in relation to effective antibiotic therapy.

\section{CASE REPORT}

A 78-year-old woman had been admitted to a peripheral hospital with shortness of breath and fatigue that had been increasing for 4 months.* Her medical history was significant for third-degree heart block, insertion of a pacemaker, rightsided heart failure, tricuspid regurgitation, coronary artery bypass grafting, and hypoglycemia. Eight days after admission to the peripheral hospital, the patient was transferred to the critical care unit of Sunnybrook Health Sciences Centre, Toronto, Ontario.

At the peripheral hospital (before the patient was transferred), initial chest radiography revealed a large right pleural effusion. At that time, the patient was afebrile, and she was hemodynamically stable. However, the white blood cell count was elevated to $19 \times 10^{9} / \mathrm{L}$ (normal range $4 \times 10^{9}$ to $11 \times 10^{9} / \mathrm{L}$ ). Blood samples were drawn for culture, and empiric therapy with levofloxacin was started for presumed pneumonia. Within $24 \mathrm{~h}$, the patient became hemodynamically unstable as she went into shock; her systolic blood pressure dropped below $80 \mathrm{~mm} \mathrm{Hg}$, and her oxygen saturation decreased to $70 \%-80 \%$ on room air. Ventilation and insertion of a chest tube were required to relieve the pleural effusion. Culture of the blood samples yielded positive results for $L$ monocytogenes sensitive to penicillin, but minimum inhibitory concentrations were not determined. At that point, the patient was switched from levofloxacin to piperacillin- tazobactam. Transthoracic echocardiography at the peripheral hospital revealed tricuspid regurgitation with vegetation and irregularities along the pacemaker wire in the right atrium. By 8 days after the original admission, oliguric renal failure had developed, and the patient's serum creatinine was rising. The patient needed continuous venovenous hemodialysis

*The authors' institution does not require informed consent to be obtained for the publication of case reports from which all patient identifiers have been removed. 
(CVVHD), but this procedure was not available at the peripheral hospital, and she was therefore transferred to the authors' facility (Sunnybrook Health Sciences Centre).

Upon arrival at the author' facility, samples (blood and bronchoalveolar lavage) were obtained for culture, including testing for Mycobacterium spp and fungi, and transthoracic echocardiography was performed. All cultures at this facility yielded negative results. Endocarditis due to $L$ monocytogenes was diagnosed on the basis of the results of blood culture and echocardiography obtained at the peripheral hospital, as well as the patient's clinical presentation. At the time of admission to the authors' facility, the patient's white blood cell count was $12.7 \times 10^{\circ} / \mathrm{L}$, and her serum creatinine was $475 \mu \mathrm{mol} / \mathrm{L}$. Estimated creatinine clearance was $9 \mathrm{~mL} / \mathrm{min}$ with no urine output, and CVVHD was initiated. The patient was switched from piperacillin-tazobactam to ampicillin $2 \mathrm{~g}$ IV every $4 \mathrm{~h}$ and gentamicin $140 \mathrm{mg}(2 \mathrm{mg} / \mathrm{kg})$ IV every $24 \mathrm{~h}$. The gentamicin was dosed for synergistic activity with the ampicillin, with a target peak concentration of $3-5 \mathrm{mg} / \mathrm{L}$ and target trough concentration of less than $2 \mathrm{mg} / \mathrm{L}$. With the stated dosage, the gentamicin peak and trough concentrations were within target range, and the planned duration of antimicrobial therapy (for both ampicillin and gentamicin) was 6 weeks. The findings on repeat blood culture were all negative, and the findings of the transthoracic echocardiography at the authors' facility were inconclusive, although this imaging did reveal irregularities on the pacemaker wires; as such, an infection at the pacemaker site could not be ruled out. The patient was not a candidate for surgery to remove the pacemaker because of her hemodynamic instability and need for dialysis. The patient died 14 days later.

\section{DISCUSSION}

Listeria monocytogenes only rarely causes infection at pacemaker sites. A MEDLINE search from 1950 to March 2010 did not yield any reports of pacemaker infections caused by this organism.

Pacemaker infections have been reported in $0.13 \%$ to $19.9 \%$ of patients. ${ }^{6}$ In cases of infection involving pacemakers, removal of the medical device is preferred when feasible, because morbidity and mortality are higher without removal. ${ }^{6}$ In addition, antimicrobial therapy directed at the organism that is causing the infection should be initiated. ${ }^{6}$ If no organism is identified and infection at the pacemaker site is suspected, empiric treatment with broad-spectrum therapy is crucial. The duration of treatment must be individualized, according to response. ${ }^{6}$ If the pacemaker is removed, the minimum recommended duration of therapy is 14 days after removal of the device or the first negative blood culture result, whichever occurs later. ${ }^{6}$ However, 4 weeks of antimicrobial therapy has been recommended for patients with Staphylococcus aureus bacteremia due to an infected device or in the presence of vegetations. ${ }^{6}$ Information about the optimal duration of therapy for patients whose pacemakers cannot be removed is currently lacking. However, the minimal duration of therapy should be 4 to 6 weeks, as for endocarditis. Because $L$ monocytogenes does not commonly cause this type of infection and because surgery was not feasible for the patient described here, it was decided to treat the infection as a case of endocarditis, with a minimum duration of therapy of 6 weeks. Endocarditis caused by $L$ monocytogenes is the focus of the remainder of this discussion.

Endocarditis due to $L$ monocytogenes is a subacute disease that may present with weakness, dyspnea, and cardiac murmur. ${ }^{4,5,7}$ Acute congestive heart failure may be present in $50 \%$ of patients. ${ }^{7}$ Listeria monocytogenes is identified as the agent causing endocarditis through blood culture. ${ }^{4,5,7}$ Patient populations at risk of $L$ monocytogenes endocarditis include neonates, pregnant women, elderly patients, individuals with impaired cell-mediated immunity, and patients with a history of rheumatic heart disease, hypertrophic cardiomyopathy, mitral prolapse, or ischemic cardiomyopathy ${ }^{4,8}$

A higher incidence of $L$ monocytogenes endocarditis has been reported among males than females (1.6:1). ${ }^{2}$ In a review of 68 cases of $L$ monocytogenes endocarditis, $60 \%$ of the patients had some type of valve alteration, and $33 \%$ had a prosthetic valve. $^{2}$ According to a world literature review, ${ }^{1}$ patients with infection of a native valve had a lower mortality rate (about $31 \%$ ) than those with infection of a prosthetic valve (about $41 \%)$. Survival rates were similar for patients who received only antibiotic treatment and those who received antibiotic treatment combined with surgery, and there seems to be no consensus as to whether surgical treatment for $L$ monocytogenes endocarditis presents extra benefit beyond that of antibiotic treatment alone. ${ }^{1}$ Spyrou and others ${ }^{1}$ recommended that surgery be reserved for patients in whom the organism is resistant to antibiotics and where significant disruption of the valve apparatus has occurred.'

Antibiotics used in the case reports reviewed by Spyrou and others ${ }^{1}$ included penicillin plus streptomycin, for which the survival rate was $73 \%$ ( 8 of 11 ), and penicillin plus an unidentified aminoglycoside, for which the survival rate was 63\% (10 of 16). The survival rate for the latter group was similar to that of penicillin alone (64\% [7 of 11]). ${ }^{1}$ Although experimental data have shown synergism between ampicillin or penicillin and aminoglycoside against $L$ monocytogenes, published comparative studies are lacking, and case reports have not demonstrated an increase in survival with combination therapy. ${ }^{1}$

In a review by Guerrero and others, ${ }^{4} 68$ patients were reported to have $L$ monocytogenes endocarditis, and the majority of the patients $(60 \%)$ had an underlying valve disorder. Fortyone percent of these patients had chronic debilitating conditions (diabetes mellitus, cirrhosis, alcoholism, or impairment of cell-mediated immunity) and 16\% had severe immunocompromise because of solid organ transplantation, leukemia or lymphoma, corticosteroid therapy, hemodialysis, or AIDS. ${ }^{4}$ Listeria monocytogenes endocarditis occurred on abnormal native 
$(66 \%)$ or prosthetic $(34 \%)$ valves, mainly on the aortic valve ( $27 \%$ of native valves versus $35 \%$ of prosthetic valves), the mitral valve (16\% of native valves versus $52 \%$ of prosthetic valves), or both the aortic and mitral valves (31\% of native valves versus $13 \%$ of prosthetic valves); it was rarely seen on the tricuspid valve ( $4 \%$ of native valves versus $0 \%$ of prosthetic valves). ${ }^{4}$ Penicillin and ampicillin (as monotherapy) are the drugs most frequently used to treat this type of endocarditis, but some strains of the bacterium may exhibit tolerance to these antibiotics, defined as a decreased rate and extent of killing. Monotherapy with penicillin had a success rate of 71\% (10 of 14 patients), whereas treatment with penicillin plus gentamicin or with ampicillin plus gentamicin yielded a success rate of $66 \%$ (23 of 35 patients). ${ }^{4}$ There were no baseline differences in the patients. The combination of vancomycin plus gentamicin was successful for 4 patients ${ }^{4}$ (although the total number of patients treated with this combination was not reported, and thus the success rate cannot be calculated). The rationale for adding an aminoglycoside to penicillin is the synergistic activity that has been observed between these 2 types of antibiotics both in vitro and in vivo. ${ }^{4}$ However, this synergism has not always been observed in vivo, and retrospective clinical studies have not shown better results with this combination than with other combinations. ${ }^{4}$ Overall mortality for the cases reviewed by Guerrero and others ${ }^{4}$ was reported as $35.3 \%$, and most deaths occurred before 1985 . The annual mortality rate decreased by $12 \%$ after 1985 , possibly because of the increased use of surgery or greater appropriateness of surgery (valve replacements). ${ }^{4}$

More recently, a case report described the use of linezolid therapy for a patient with $L$ monocytogenes endocarditis that did not improve with 2 weeks of vancomycin therapy. ${ }^{2}$ However, linezolid is not considered optimal for endocarditis because it is only bacteriostatic (not bactericidal) against $L$ monocytogenes and is associated with an increased risk of toxic effects with long-term use (i.e., more than 2 weeks). ${ }^{2}$ Its place in the treatment of infective endocarditis remains unknown.

In selecting the appropriate antibiotic therapy for $L$ monocytogenes infections, it is important to note the bactericidal activity of the various antimicrobial agents, as well as the reported resistance. The antibiotics with bactericidal activity against $L$ monocytogenes are penicillin, ampicillin, amoxicillin, vancomycin, and co-trimoxazole. ${ }^{5}$ High-dose $\beta$-lactam antibiotics achieve this effect by reducing the numbers of intracellular $L$ monocytogenes organisms. The antibiotics with bacteriostatic activity against $L$ monocytogenes are imipenem, erythromycin, tetracycline, and ciprofloxacin. ${ }^{5}$ Antibiotic resistance has only rarely been reported with tetracycline and erythromycin. ${ }^{5}$ The mode of resistance is enterococcal plasmids encoding resistance determinants, which are easily transferred in vitro to the bacteria. Most strains of $L$ monocytogenes have high resistance to cephalosporins (especially cefotaxime). ${ }^{5}$ Resistance to this drug stems from a lack of appropriate penicillin-binding protein in the cytoplasmic membrane. ${ }^{5}$

\section{CONCLUSIONS}

Even with appropriate medical and surgical management, $L$ monocytogenes endocarditis is associated with a high mortality rate. ${ }^{1}$ Treatment options include penicillin or ampicillin alone, penicillin or ampicillin in combination with gentamicin, or, in cases of allergy to penicillin, vancomycin plus gentamicin. ${ }^{1,4}$ Because of the high mortality rate, as well as the in vivo and in vitro data available for this organism, it is important to be aggressive with antibiotic treatment, optimizing the dosing and considering combination therapy with gentamicin, at synergistic dosing to achieve peak concentrations between 3 and $5 \mathrm{mg} / \mathrm{L}$. The usual duration of treatment for endocarditis should be 4 to 6 weeks. The response to therapy should be monitored in terms of clinical resolution of signs and symptoms, as well as appropriate antibiotic levels for efficacy and avoidance of toxic effects when aminoglycosides are used.

\section{References}

1. Spyrou N, Anderson M, Foale R. Listeria endocarditis: current management and patient outcome-world literature review. Heart 1997; 77(4):380-383.

2. Antolin J, Gutierrez R, Segoviano R, López R, Ciguenza R. Endocarditis due to Listeria: description of two cases and review of the literature. Eur J Intern Med 2008;19(4):295-296.

3. Chief Medical Officer of Health's report on the management of the 2008 listeriosis outbreak in Ontario. Toronto (ON): Ministry of Health and Long-Term Care; 2009 Apr [cited 2009 Nov 12]. Available from www.health.gov.on.ca/english/public/pub/disease/listeria/listeriosis_ outbreak_rep.pdf

4. Guerrero MLF, Rivas P, Rábago R, Núnez A, de Górgolas M, Martinell J. Prosthetic valve endocarditis due to Listeria monocytogenes. Int J Infect Dis 2004;8(2):97-102.

5. Hof H, Nichterlein T, Kretschmar M. Management of listeriosis. Clin Microbiol Rev 1997;10(2):345-357.

6. Baddour LM, Bettmann MA, Bolger AF, Epstein AE, Ferrieri P, Gerber MA, et al. Nonvalvular cardiovascular device-related infections. Circulation 2003;108(16):2015-2031.

7. Doganay M. Listerosis: clinical presentation. FEMS Immunol Med Microbiol 2003;35(3):173-175.

8. Brouqui P, Raoult D. Endocarditis due to rare and fastidious bacteria. Clin Microbiol Rev 2001;14(1):177-207.

Claudia Summa, BScPhm, ACPR, is a Clinical Pharmacist - General Internal Medicine, Department of Pharmacy, University Health Network Toronto General Hospital, Toronto, Ontario.

Sandra A N Walker, BSC, BSCPhm, ACPR, PharmD, FCSHP, is an Associate Professor (status only) with the Leslie Dan Faculty of Pharmacy, University of Toronto, and Clinical Coordinator - Infectious Diseases, Department of Pharmacy, Sunnybrook Health Sciences Centre, Toronto, Ontario.

\section{Address correspondence to: \\ Claudia Summa \\ Department of Pharmacy \\ University Health Network - Toronto General Hospital \\ 200 Elizabeth Street \\ Toronto ON M5G 2C4}

e-mail: Claudia.Summa@uhn.on.ca 